Paul V. Tozzi, Christian M. Wisniewski, Nicholas J. Zalewski, Mariano J. Savelski, C. Stewart Slater* and Frank A. Richetti

\title{
Life cycle assessment of solvent extraction as a low-energy alternative to distillation for recovery of $\mathrm{N}$-methyl-2-pyrrolidone from process waste
}

https://doi.org/10.1515/gps-2017-0030

Received February 22, 2017; accepted July 27, 2017; previously published online September 11, 2017

\begin{abstract}
This case study investigates low-energy alternatives to distillation for the recovery of N-methyl2-pyrrolidone (NMP) from solvent waste in the manufacture of resin precursors. Evaluation includes environmental and economic life cycle assessment of solvent recovery and reuse using batch distillation, solvent extraction, and solvent extraction at a sister plant. Solvent extraction at the sister plant involved shipment of hazardous waste to a facility with existing solvent recovery equipment and lower purity standards for NMP. The amount of processing required to recover useful NMP is reduced, increasing reductions in life cycle emissions and damages to the environment. Extraction at the sister plant recovered $98 \%$ of the NMP at a purity of $97.1 \mathrm{wt} \%$ from aqueous waste containing $17 \mathrm{wt} \%$ NMP. Extraction at the sister plant reduced total and $\mathrm{CO}_{2}$ life cycle emissions by $61 \%$ and $59 \%$, respectively. Compared to the distillation recovery alternative, extraction at the sister plant results in reductions of 32\% and $33 \%$ for total and $\mathrm{CO}_{2}$ life cycle emissions, respectively. Annual operating costs were reduced by $80 \%$, with no capital investment, due to utilization of existing equipment. This resulted in a 10-year net present value (NPV) of $\$ 4.20$ million, whereas distillation resulted in a 10-year NPV of \$3.12 million.
\end{abstract}

Keywords: dipolar aprotic solvent; life cycle assessment; N-methyl-2-pyrrolidone; solvent extraction; solvent recovery.

\footnotetext{
*Corresponding author: C. Stewart Slater, Department of Chemical Engineering, Rowan University, 201 Mullica Hill Road, Glassboro, NJ 08028, USA, e-mail: slater@rowan.edu

Paul V. Tozzi, Christian M. Wisniewski, Nicholas J. Zalewski and Mariano J. Savelski: Department of Chemical Engineering, Rowan University, 201 Mullica Hill Road, Glassboro, NJ 08028, USA Frank A. Richetti: DuPont Corporation, 250 Cheesequake Road, Parlin, NJ 08859, USA
}

\section{Introduction}

The objective of the following case study is to evaluate and compare low-energy alternatives to distillation for the recovery of N-methyl-2-pyrrolidone (NMP) from process waste streams in the specialty chemical industry. This study takes into consideration the production scenario at DuPont Corporation's Parlin, NJ plant, where NMP is used as the reaction medium in the manufacture of resin precursors. The use of NMP as a reaction medium stems from its thermal stability, dipolar aprotic properties, and ability to produce high-molecular weight resin precursor [1]. A significant amount of NMP solvent waste is generated as a result of the process in a washing step where NMP and other impurities are removed from the resin precursor product. Low-energy recovery techniques were investigated, building upon the investigations of Pastore et al. [2] in which a recovery system using distillation was proposed. Distillation is the most widely used solvent separation process in the chemical industry, accounting for 95\% of all separations [3]. Additionally, distillation accounts for $35 \%$ of the total energy expenditure in the chemical manufacturing industry [4].

The manufacture of resin precursors shares many characteristics with other manufacturing operations in the specialty and fine chemical industries. Processes in these industries are typically operated in batch mode, and products are created in a stepwise fashion, rather than a continuous one. Some such steps include reactions or separation and purification operations. One major drawback of this style of production is that at every step, there could be inefficiencies in chemical, water, and energy use [5]. Over the course of the entire manufacturing process, these inefficiencies are compounded, resulting in high waste per unit product. The specialty chemical sector reported 2.2 billion $\mathrm{kg}$ of waste to the Toxics Release Inventory (TRI) in 2014 [6]. Waste generated in the specialty chemical sector is primarily organic solvent waste, with some of the highest waste generation per unit product, ranging from 5 to $50 \mathrm{~kg}$ waste $/ \mathrm{kg}$ product [7]. The most common organic solvents used in the specialty chemical industry include 
toluene, methanol, 1,2-dichloroethane, dichloromethane, xylene, $\mathrm{n}$-hexane and other toxic and hazardous solvents like NMP [6].

During their life cycle, toxic solvents impact the environment outside of their use in manufacturing plants. The production and disposal of these solvents also have significant impacts on the environment [8]. In the manufacturing of the average solvent, for every kilogram of solvent produced, about $2 \mathrm{~kg}$ of emissions are released. The same is true for disposal: incineration of $1 \mathrm{~kg}$ of solvent produces slightly over $2 \mathrm{~kg}$ of emissions [9]. These life cycle emissions have detrimental effects on air, water and land. Emissions include greenhouse gases, ozone-depleting substances, volatile organic compounds, sulfur and nitrogen oxides and aqueous organic matter [10]. Reduction in the use of virgin solvents is required in order reduce their respective impact on the environment [9, 11, 12]. Use of less harmful solvents (based on safety and environmental factors) can reduce the environmental impact [13], but separation techniques such as distillation and pervaporation can be used to recover and reuse the spent solvent [8] which has been shown to be more cost effective while comparably reducing the environmental impact [2].

Industrial use of NMP comprises production of basic organic chemicals, specialty/fine chemicals, pharmaceutical compounds, and polymeric materials [6]. The widespread use of NMP is due in part to its ability to dissolve materials that will not dissolve in many other solvents. NMP is also valuable in processes due to its dipolar aprotic properties [14]. In polymer manufacturing, NMP is used in the synthesis and processing of polyamides, polyimides, polyethersulfones, polyarylene ethers and polyurethanes [15-18]. Use of NMP for the manufacture of these polymers presents a problem because NMP is not consumed in the process and leaves the process as waste [19]. The TRI shows that NMP waste generation in the polymer manufacturing industry has more than doubled over the past decade [6]. Even with the increase in NMP usage in this industry, there has been only one published environmental and economic case study investigating the recovery of NMP from a polymer manufacturing process [2]. Although useful industrially, production and disposal of NMP negatively impacts the environment. Furthermore, NMP is suspected to be a reproductive and developmental toxin [20]. Disposal of aqueous NMP typically involves incineration, which requires additional fuel and creates $\mathrm{NO}_{X}$ emissions [14, 21].

In many of the industrial processes in which dipolar aprotic solvents like NMP are used, there is no suitable solvent replacement [14, 21-23]. As a result, NMP use cannot be reduced easily in the specialty chemical sector. Typically, the spent NMP is disposed of in the form of aqueous waste, like in this case study. Rarely is the NMP recovered because separation is believed to be both difficult and costly [14, 21, 23-25]. Pastore et al. [2] explored the capabilities of distillation as a method for recovering NMP from the waste generated from DuPont's resin precursor process. Though this investigation proved distillation's feasibility as a solvent recovery technique, it was clear that use of a less energy intensive separation technique or solvent reuse option would show greater reductions in emissions and operating costs. This particular study fills the need, as stated by the National Research Council, to promote "more energy and cost efficient chemical separations, especially effective alternatives to distillation" [26].

The primary focus of this study is solvent extraction as an alternative to distillation, but prior to performing a rigorous case study analyzing solvent extraction, other low-energy separations were explored alongside solvent extraction, including dividing wall columns (DWCs), heat pump assisted distillation (HPAD), vacuum distillation, pervaporation and cryogenic separation. DWCs have been shown to reduce both energy expenditure and capital cost of the process equipment by up to $30 \%$ [27]; however, appropriately designing a DWC for NMP recovery proved too challenging to continue investigation [28]. The HPAD and vacuum distillation were shown to reduce energy usage $[29,30]$, but neither outperformed solvent extraction in this case. A pervaporative recovery system was designed using lab-scale data for dehydration of aqueous NMP waste [31] and organic selective separation of a similar dipolar aprotic solvent [32]; it is not clear whether the use of lab-scale data can accurately reflect larger scale systems; preliminary scale-up calculations predicted a membrane size which would be cost-prohibitive. Therefore, further investigation of pervaporation was not considered. Cryogenic separation was not further investigated due to lack of available data for NMP purification [30]. Solvent substitutes for NMP were also explored by Pastore et al. [2]; dimethylsulfoxide and sulfolane were considered as solvent substitutes for NMP. Solvent substitution did not provide significant reductions in environmental impact when a recovery system was integrated [2]. Solvent extraction was ultimately chosen as the focal point for this research because it can be implemented more easily than other alternatives that exhibited various limitations, and as will be discussed, existing use data exists.

The potential impact of this study is widespread. Like the results presented by Pastore et al. [2], the results presented here show how solvent recovery can significantly reduce life cycle emissions and operating expenses for processes using solvents such as NMP. However, unlike the study by Pastore et al. [2], this study also shows how 
existing solvent recovery operations that utilize distillation can be improved upon by considering low-energy alternatives and other options for solvent reuse beyond the particular plant site.

\section{Materials and methods}

In order to maintain consistency with the previous study, the same evaluation techniques were used to quantify differences between NMP recovery designs and the base case. Life cycle assessment (LCA) techniques were used to determine the environmental and economic effects of the base case and several green engineering alternatives. Design and simulation of green engineering alternatives was performed using Aspen Plus ${ }^{\circledR}$ v8.6 (Aspen Technology, Inc., Bedford, MA, USA). Aspen Plus simulations provide detailed reports containing values for flow rates, composition and utility needs. After a rigorous design and simulation procedure, data from Aspen Plus were used to perform an LCA for each of the designs. These LCAs were used to identify differences among the base case and alternatives. Aspen Capital Cost Estimator, a tool available within Aspen Plus, was used to determine the installed capital cost of the alternatives. The major goal of this analysis was to improve upon the design identified by the previous study as the most promising: NMP recovery using batch distillation. The previous study showed that batch distillation was an attractive alternative to the base because the operating costs and life cycle emissions were significantly reduced, but further reductions appeared attainable.

The system boundaries of the LCA include the production of ultrapure water used to wash the resin precursor, as well as the washing step itself, in which the resin precursor is precipitated and subsequently washed, removing NMP and other impurities. These boundaries were chosen in order to focus on the effects of solvent use and recovery. This methodology is consistent with several other investigations that use LCA techniques to analyze solvent use in the chemical and pharmaceutical manufacturing industries $[2,9,11$, 12]. The green engineering alternatives presented in this case study are included within the system boundaries. Chemicals entering or leaving the system in the base case or in each of the presented alternatives are accounted for in the LCAs. For chemicals entering the system, the environmental impact associated with their production is accounted for. For chemicals leaving the system, the environmental impact associated with their disposal is accounted for. Impact attributed to utility use within the boundaries is also taken into account. Utilities include electricity, steam, and cooling water.

For each of the cases investigated, three LCAs were performed. This included two environmental LCAs and an economic LCA. One of the environmental LCAs was based on life cycle emissions, and the other was based on the Hierarchist perspective of the ReCiPe method [33]. ReCiPe is an LCA methodology that gets its namesake from the major contributors: RIVM and Radboud University, CML and PRé Consultants. The Hierarchist perspective of this method is based on the most common policy principles with regard to time and other issues. The economic LCA was based on life cycle operating costs of the base case and green engineering alternatives. Annualized usage and the life cycle inventories (LCI) for raw materials, waste, and utilities were used to calculate the LCAs of each case. The LCIs for raw materials, waste, and utilities were determined on a $1 \mathrm{~kg}$ or $1 \mathrm{MJ}$ basis (Table 1). LCIs for the emissions LCA included emissions to the air, water, and soil, as well as $\mathrm{CO}_{2}$ emissions. The ReCiPe endpoint method used LCIs that included single scores for the human health, ecosystems, and resources categories. Economic LCIs included raw material purchase and disposal costs, along with utility costs.

Environmental LCIs were obtained from SimaPro ${ }^{\circledR}$ v8 (PRe Sustainability, Amersfoort, The Netherlands) and economic LCIs were either provided by DuPont of found in literature [34-36]. LCIs were found for the manufacture of NMP, hydroxyethyl methacrylate (HEMA), trifluoroacetic acid (TFA), $\mathrm{HCl}, \mathrm{Ca}(\mathrm{OH})_{2}$, chloroform and ultrapure water; the generation of steam and electricity; and the disposal of hazardous waste and nonhazardous wastewater. Disposal of hazardous waste was modeled as incineration and disposal of nonhazardous wastewater was modeled as being sent to a municipal wastewater treatment facility.

\subsection{Base case: incineration}

The resin precursor process manufactures liquid polyimide (PI) and polybenzoxazole (PBO) precursors that are cured by DuPont's

Table 1: Summary of LCls used in the analysis of the base case and recovery options.

\begin{tabular}{|c|c|c|c|c|c|c|}
\hline Input/output (basis) & $\begin{array}{r}\mathrm{CO}_{2} \text { emissions } \\
(\mathrm{kg})\end{array}$ & $\begin{array}{l}\text { Total life cycle } \\
\text { emissions (kg) }\end{array}$ & $\begin{array}{r}\text { Human health } \\
(\mathrm{mPt})\end{array}$ & $\begin{array}{r}\text { Ecosystems } \\
(\mathrm{mPt})\end{array}$ & $\begin{array}{r}\text { Resources } \\
(\mathrm{mPt})\end{array}$ & Cost (\$) \\
\hline NMP (1 kg) & 3.72 & 4.22 & 146 & 76.9 & 215 & 4.30 \\
\hline Ultrapure water (1 kg) & 0.00913 & 0.0115 & 0.315 & 0.172 & 0.229 & 0.0135 \\
\hline Electricity (1 MJ) & 0.0957 & 0.111 & 4.26 & 1.91 & 4.78 & 0.0314 \\
\hline Steam (1 MJ) & 0.0667 & 0.0671 & 2.01 & 1.26 & 3.38 & 0.00682 \\
\hline Hazardous waste disposal (1 kg) & 0.193 & 0.217 & 5.57 & 3.58 & -8.71 & 0.531 \\
\hline Wastewater disposal (1 kg) & 0.0275 & 0.0280 & 0.891 & 0.518 & 0.227 & 0.0105 \\
\hline HEMA (1 kg) & 5.59 & 5.78 & 231 & 122 & 326 & 2.34 \\
\hline TFA (1 kg) & 4.16 & 4.80 & 247 & 88.2 & 204 & 0.278 \\
\hline $\mathrm{HCl}(1 \mathrm{~kg})$ & 1.27 & 1.50 & 70.3 & 26.2 & 48.9 & 0.278 \\
\hline $\mathrm{Ca}(\mathrm{OH})_{2}$ for neutralization $(1 \mathrm{~kg})$ & 0.740 & 0.751 & 22.6 & 13.8 & 11.0 & 0.248 \\
\hline Chloroform (1 kg) & 1.77 & 2.02 & 578 & 149 & 194 & 0.805 \\
\hline Transportation of hazardous waste $(1 \mathrm{t} \mathrm{km})$ & 0.0866 & 0.0975 & 8.93 & 3.63 & 7.73 & 0.180 \\
\hline
\end{tabular}


customers to form PI and PBO resins. PI and PBO resins can be used in microelectronics, microelectromechanical systems and other applications [37]. The Parlin plant currently handles the waste by disposing it off-site for hazardous waste incineration. This approach is expensive, and even with heat recovery, generates significant life cycle emissions. The waste generated equates to 1,077,000 kg/year of waste composed of $81.5 \%$ water, $17 \%$ NMP, $0.5 \%$ HEMA, $0.5 \%$ TFA and $0.5 \% \mathrm{HCl}$. Additionally, 1,209,000 kg/year of total life cycle emissions are generated, $89 \%$ of which are $\mathrm{CO}_{2}$ emissions. NMP manufacture and hazardous waste disposal account for $64 \%$ and $19 \%$ of the total life cycle emissions, respectively. The life cycle operating cost of the base case is 1,466,000 \$/year. NMP manufacture and hazardous waste disposal account for $54 \%$ and $39 \%$ of the life cycle operating cost, respectively. More details regarding the base case can be found in Pastore et al. [2].

\subsection{NMP recovery: distillation}

After investigating several NMP recovery options, Pastore et al. [2] determined that distillation would serve best as the recovery method for this application. The proposed distillation recovery operation utilizes a single distillation column for two batch distillation steps. The first removes most of the water and acids from the base case's hazardous waste, which is then neutralized and disposed of as nonhazardous wastewater. The second step then removes the HEMA from the remaining waste. The HEMA is disposed of as hazardous waste, and the remainder is $99.97 \%$ pure NMP, ready to be reused in the resin precursor manufacturing process. The distillation system recovers $94.7 \%$ of the NMP from the hazardous waste. Relevant flowrates and compositions for this recovery system can be found in Table 3.

Recovery of NMP using distillation reduces total life cycle emissions by $44 \%$ and reduces life cycle $\mathrm{CO}_{2}$ emissions by $40 \%$. The ReCiPe endpoint single scores for recovery using distillation in the human health, ecosystems and resources categories were reduced by $46 \%, 42 \%$ and $27 \%$, respectively, when compared to the base case. The life cycle operating cost of the base case is reduced by $1,217,000$ \$/year or $83 \%$ when NMP is recovered using distillation. The installed capital cost of the equipment for this recovery method is $\$ 1,498,000$, according to the Aspen Capital Cost Estimator. Using the economic analysis described by Pastore et al. [2], distillation saves $\$ 1,750,000$ over the first 5 years after instillation and $\$ 3,128,000$ over the first 10 years (Table 2). Other alternatives that were considered as well as a more detailed description of the distillation process can be found in Pastore et al. [2].

\subsection{NMP recovery: on-site solvent extraction}

As stated in the previous case study, distillation can be energy intensive, which can negatively affect operating costs and environmental impact [14, 21, 23, 31,38]. This concern is particularly applicable in this case due to NMP and HEMA being high-boiling organic compounds, which indicates that large amounts of energy will be required to separate the two compounds. Similarly, the major component of the waste is water, which is a lower boiling compound than both NMP and HEMA. As a result, a large amount of energy in the form of steam is required to remove the water from the waste in the first distillation step of the proposed recovery method. In fact, $87 \%$ of the steam used in the distillation recovery scheme goes towards removing the water.

Extraction as a low-energy alternative to distillation has been explored for use in biofuels, for separating high-boiling organic compounds from fermentation broths with a high water content [39, 40]. Again, the purification of aqueous mixtures with low mass fractions of high-boiling organic material is both energy and cost intensive. In this case, a high-boiling organic, namely NMP, is to be removed from waste with a high water content. Research performed in the field has proven the efficacy of extraction for similar aqueous-organic separations, but at the time of the previous study [2], investigation of extraction was not considered, due to limited data for NMP extraction from aqueous mixtures [23, 38]. A laboratory study by Yang and Zhu [41] tested several extracting solvents and showed chloroform performed best for recovering NMP from solvent waste. These claims were independently evaluated using their Hansen Solubility Parameters [42] and process simulation in Aspen Plus. The results of the study were confirmed, and chloroform was chosen as the extracting solvent for future process simulations. Chloroform not only showed high NMP recovery, but was also easy to separate and recycle from the extract stream using distillation. Selecting a solvent with such properties is crucial for designing an energy efficient extraction-based solvent recovery process. Additionally, DuPont uses chloroform at another facility to recover large quantities of NMP from solvent waste, further supporting the choice of extracting solvent.

\section{Results and discussion}

\subsection{NMP recovery: on-site solvent extraction}

The extraction process for recovering NMP from the hazardous solvent waste is shown in Figure 1. As in the distillation design, the waste collects in a holding tank, prior to treatment. Once enough waste is collected, it is preheated before being fed to an extraction column along with recycled extracting solvent and make-up extracting solvent. The feed is preheated because the recycled extracting solvent is at a high temperature after leaving the first

Table 2: Capital, operating costs; net present value (NPV) after 5 and 10 years.

\begin{tabular}{lrrrr}
\hline Scenario & Capital cost (\$) & Operating cost (\$\$year) & NPV after 5 years (\$) & NPV after 10 years (\$) \\
\hline Distillation: Parlin & $1,498,000$ & 249,000 & $1,750,000$ & $3,128,000$ \\
Extraction: Parlin & $2,062,000$ & 224,000 & $1,334,000$ & $2,762,000$ \\
Extraction: Sister & - & 289,000 & $2,923,000$ & $4,198,000$ \\
\hline
\end{tabular}




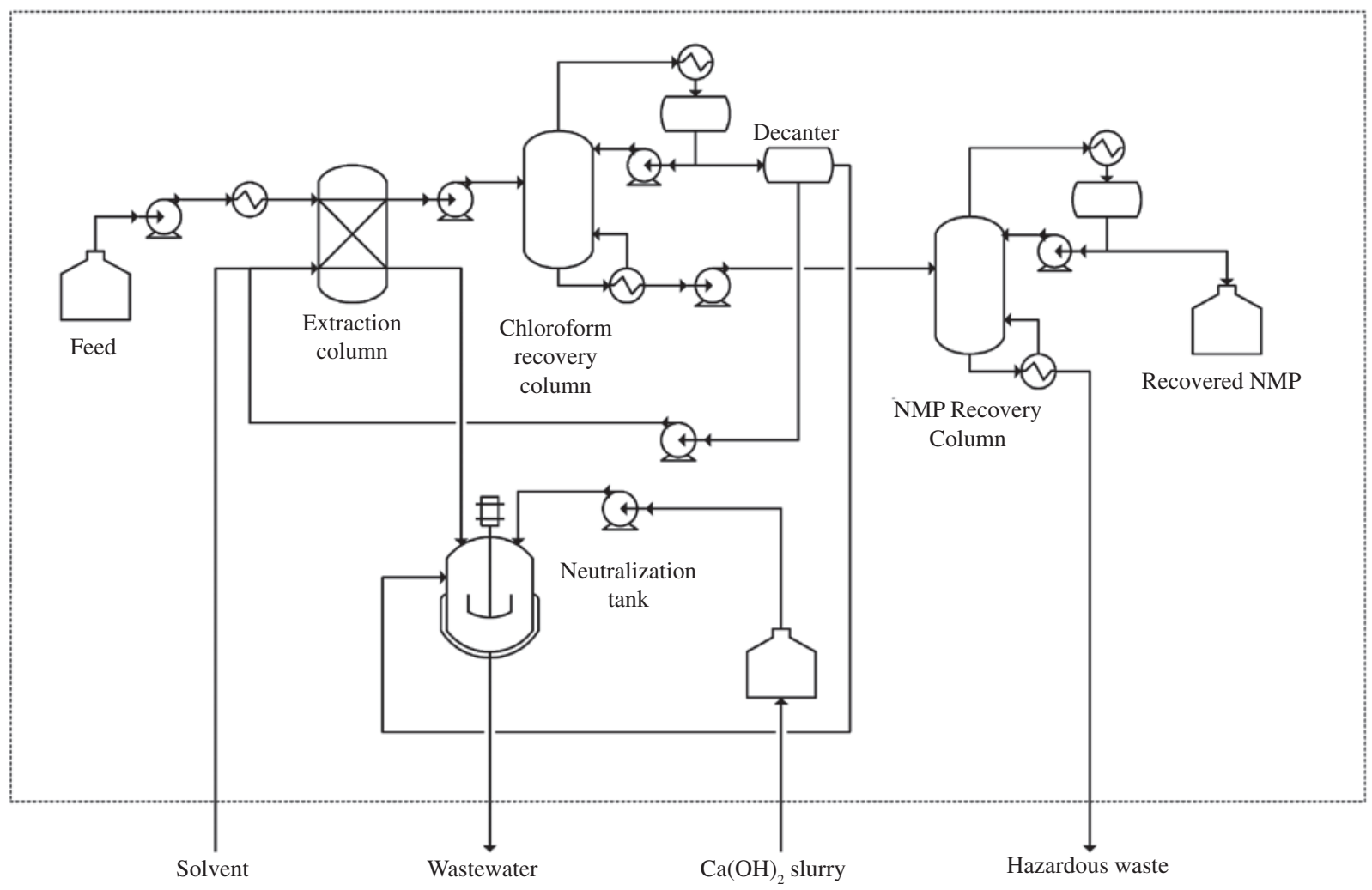

Figure 1: Extraction recovery system flow diagram.

distillation column. Process simulations performed in Aspen Plus showed that a feed at room temperature resulted in a higher water content in the extract. Other alternative methods for heating and cooling particular streams resulted in higher operating costs and emissions.

The raffinate, which is composed of most of the water and acidic components of the waste, is sent to a neutralization tank where it is treated before being disposed of as nonhazardous wastewater. The extract contains the extracting solvent, NMP and HEMA. The extract is sent to a distillation column to recover the extracting solvent. The distillate is mostly chloroform, with a small amount of water, which is removed using a decanter. The water removed is sent to the previously mentioned neutralization tank, and the recovered extracting solvent is reused in the extraction process. The bottom product of the first distillation column is a mixture of NMP and HEMA. This mixture is sent to a second distillation column where these components are separated. The HEMA is treated as a hazardous waste and sent for incineration. The recovered NMP is stored in a holding tank, ready to be reused in the resin precursor manufacturing process. The extraction system shown in Figure 1 recovers $96.2 \%$ of the NMP from the hazardous waste at a purity of $99.98 \%$. The remainder consists of $0.014 \%$ HEMA as well as trace amounts of water, TFA, $\mathrm{HCl}$ and chloroform. Relevant flowrates and compositions for this recovery system can be found in Table 3.

Like the proposed distillation recovery system, this design also requires high-pressure steam to operate the two distillation columns. However, the necessary amount of steam is reduced by about $48 \%$. This reduction in steam usage is responsible for reductions in life cycle emissions; damage to human health, ecosystems, and resources; and operating costs. The life cycle emissions associated with the base case, distillation recovery option and on-site extraction recovery option are shown in Figure 2. The on-site extraction recovery option generates $463,000 \mathrm{~kg} /$ year of total life cycle emissions and 436,000 kg/year of $\mathrm{CO}_{2}$ life cycle emissions. These values represent a $62 \%$, and $59 \%$ reduction in total life cycle emissions and $\mathrm{CO}_{2}$ life cycle emissions, respectively, compared to the base case. Compared to the previously proposed distillation design, total life cycle emissions are reduced by $32 \%$ and $\mathrm{CO}_{2}$ life cycle emissions are reduced by $33 \%$. The NMP recovery using extraction results in life cycle emissions reductions because of reduced virgin NMP use, reduced hazardous waste disposal and reduced steam usage. The chloroform 
Table 3: Mass flowrates and weight percent compositions for select streams of recovery alternatives; lons: ions are introduced by $\mathrm{Ca}(\mathrm{OH})_{2}$ in the neutralization process.

\begin{tabular}{|c|c|c|c|c|c|c|c|c|}
\hline & \multirow{2}{*}{$\begin{array}{r}\text { Mass flowrate } \\
(\mathrm{kg} / \text { year) }\end{array}$} & \multicolumn{7}{|c|}{ Composition (wt\%) } \\
\hline & & Water & NMP & HEMA & $\mathrm{HCl}$ & TFA & Chloroform & Ions \\
\hline Solvent waste & $1,077,300$ & 81.5 & 17 & 0.5 & 0.5 & 0.5 & - & - \\
\hline \multicolumn{9}{|l|}{ Distillation: Parlin } \\
\hline Recovered NMP & 173,300 & $300 \mathrm{ppm}$ & 99.97 & $35 \mathrm{ppm}$ & - & - & - & - \\
\hline Hazardous waste & 14,500 & Trace & 63 & 37 & - & - & - & - \\
\hline Lime slurry $\left[15\right.$ wt $\left.\% \mathrm{Ca}(\mathrm{OH})_{2}\right]$ & 48,200 & - & - & - & - & - & - & - \\
\hline Nonhazardous waste & 937,600 & 98.36 & 0.05 & Trace & - & - & - & 1.6 \\
\hline \multicolumn{9}{|l|}{ Extraction: Parlin } \\
\hline Make up solvent & 6500 & - & - & - & - & - & 100 & - \\
\hline Recovered NMP & 176,400 & Trace & 99.98 & 0.014 & Trace & Trace & Trace & - \\
\hline Hazardous waste & 8800 & Trace & 38.8 & 61.2 & Trace & Trace & Trace & - \\
\hline Lime slurry $\left[15\right.$ wt $\left.\% \mathrm{Ca}(\mathrm{OH})_{2}\right]$ & 48,400 & - & - & - & - & - & - & - \\
\hline Nonhazardous waste & 947,100 & 97.4 & 0.34 & Trace & - & - & 0.72 & 1.52 \\
\hline \multicolumn{9}{|l|}{ Extraction: Sister } \\
\hline Make up solvent & 6500 & - & - & - & - & - & 100 & - \\
\hline Recovered NMP & 185,100 & Trace & 97.1 & 2.9 & Trace & Trace & Trace & - \\
\hline Lime slurry $\left[15\right.$ wt $\left.\% \mathrm{Ca}(\mathrm{OH})_{2}\right]$ & 48,398 & - & - & - & - & - & - & - \\
\hline Nonhazardous waste & 947,100 & 97.4 & 0.34 & Trace & - & - & 0.72 & 1.52 \\
\hline
\end{tabular}

NMP, N-methyl-2-pyrrolidone; HEMA, hydroxyethyl methacrylate; $\mathrm{HCl}$, hydrochloric acid; TFA, trifluoroacetic acid.

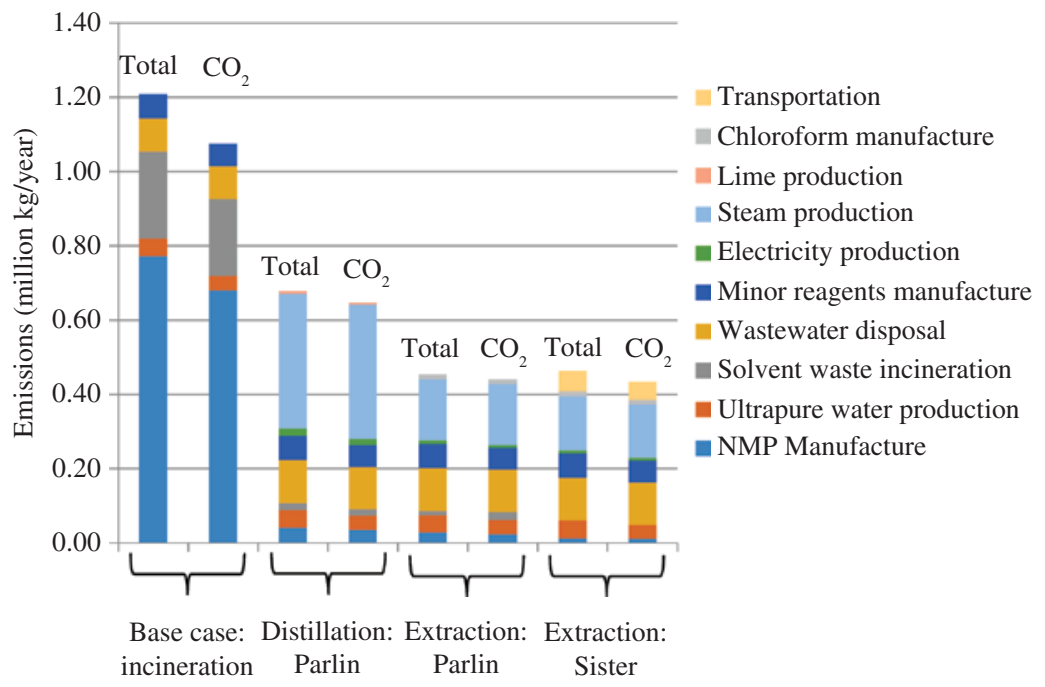

Figure 2: Life cycle emissions for base case and alternative cases.

Total, total emissions; $\mathrm{CO}_{2}$, carbon dioxide emissions.

used in the extraction process does not have a significant impact on the LCA; the design incorporates a chloroform recovery system and only therefore the make-up chloroform is accounted for in LCA calculations.

The ReCiPe endpoint single scores for the base case and recovery cases are displayed in Figure 3. Recovery of NMP using on-site extraction produces single scores for human health, ecosystems, and resources of 18,200, 9100 and 16,100 Pt/year, respectively. The NMP recovery using on-site extraction reduces the scores by 54\%, 58\% and $54 \%$ for human health, ecosystems, and resources, respectively. Compared to recovery using distillation, the scores are reduced by an additional 16\%, 27\% and 37\% for human health, ecosystems, and resources, respectively. Most of the further reductions are caused by the decrease in steam usage compared to the distillation recovery option.

The life cycle operating costs for the base case and alternative cases are displayed in Figure 4. On-site recovery 


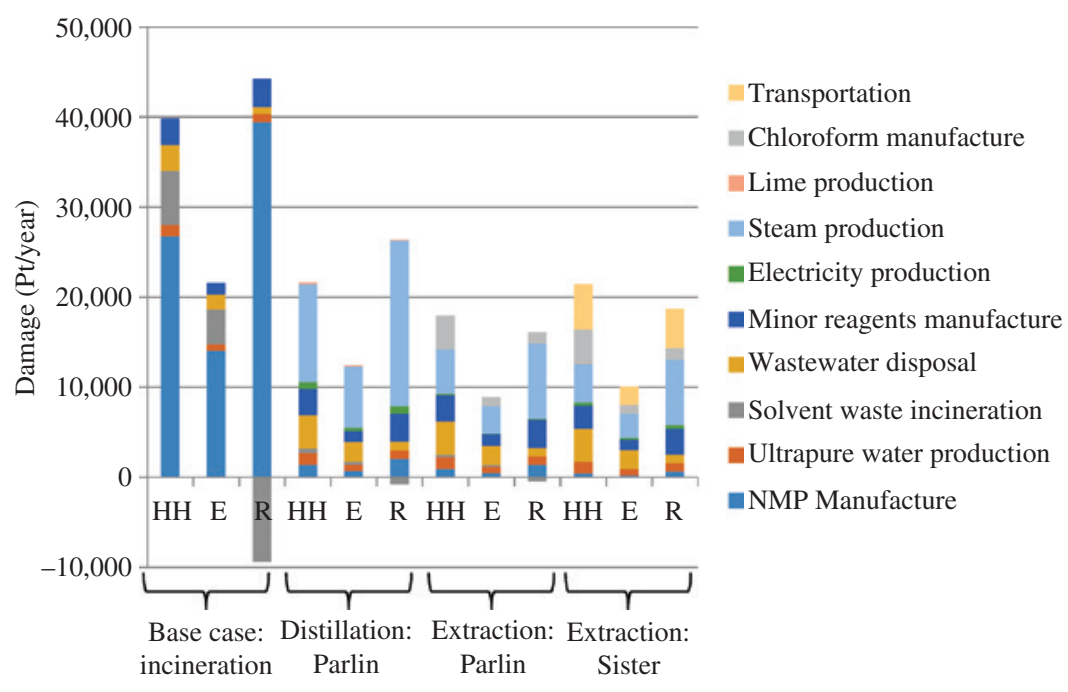

Figure 3: ReCiPe endpoint single scores for base case and alternative cases. $H H$, human health; $E$, environment; $R$, resources.

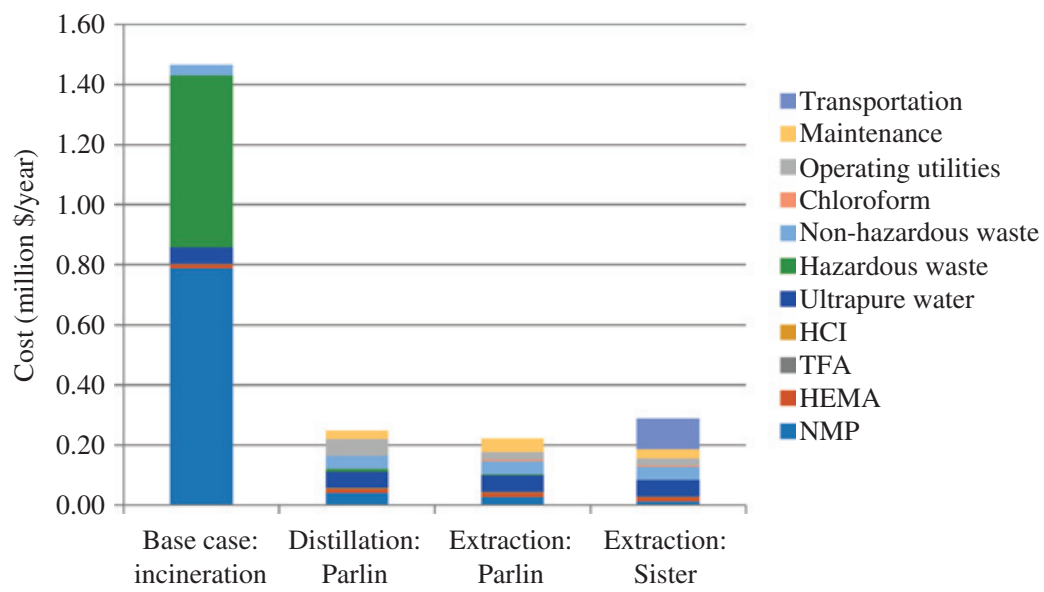

Figure 4: Life cycle operating costs for base case and alternative cases.

using extraction has a life cycle operating cost of 224,000 $\$ /$ year, resulting in a reduction of $1,242,000$ \$/year or $85 \%$. These reductions are similar to those produced by recovery using distillation, which were $1,217,000$ \$/year or $83 \%$. Again, the reduction in steam usage reduces operating costs, but a greater number of pieces of equipment increase maintenance costs. Only the make-up chloroform costs are included in the operating expenses as the boundaries include the chloroform recovery system. To better compare the economic feasibility of recovery options, a more detailed economic analysis was required. This analysis takes both operating and installed capital cost of the alternative into consideration. The details of the analysis method are discussed in detail by Pastore et al. [2]. Ultimately, the capital cost of this alternative was determined to be $\$ 2,062,000$. This, along with the savings of $1,242,000 \$ /$ year, results in 5-year net present value (NPV) of $\$ 1,334,000$ and a 10-year NPV of $\$ 2,762,000$ (Table 2). The distillation recovery method results in a 5-year NPV of $\$ 1,750,000$ and a 10-year NPV of $\$ 3,128,000$ (Table 2). In the long run, this alternative reduces emissions, and saves money even with the purchase of equipment. It reduces emissions more than the distillation recovery option, but does not save as much money over the course of 5 or 10 years.

\subsection{NMP recovery: solvent extraction and reuse at the sister plant}

Another aspect of the study was to determine if there were other DuPont facilities that used/disposed NMP and would be interested in this recovery project. One thought 
was that multiple locations with similar waste streams might have a desire to "pool" resources for a capital improvement project for a joint solvent recovery facility or the other plants might already have underutilized capacity to recover the solvent with existing extraction equipment. The TRI data for all DuPont facilities was analyzed to determine facilities of interest based on NMP disposal.

One plant (referred to as "the sister plant" from this point forward) was identified and investigated as a candidate for off-site solvent recovery and reuse. This DuPont facility produces other specialty chemicals, generates a significant amount of aqueous NMP waste, and recovers NMP for reuse on-site with an extraction process. Based on the analysis of TRI data, despite having an NMP recovery system, this plant still disposes highly contaminated NMP waste. Therefore, it was assumed that this plant has a need for more purified NMP waste, and would benefit from recovering and reusing the NMP in Parlin's waste. The amount of waste processed at this facility is very large compared to the waste generated by the Parlin facility, which is equivalent to $<1 \%$ of the other plant's waste. This indicates that processing of the Parlin waste should not have a significant impact on the other plant's day-to-day operations.

This plant has a large-scale extraction column that uses chloroform as the extracting solvent for NMP recovery. To provide a uniform method of LCA analysis, the process at the other plant was modeled as the proposed on-site design without the distillation column that further purifies the recovered NMP by removing the HEMA. The other plant uses NMP in the manufacture of specialty chemicals that do not require electronics-grade NMP, allowing for the removal of the second distillation column that separates the NMP and HEMA. The HEMA left in the recovered waste, when mixed with the waste the other plant already recovers, results in a HEMA concentration of approximately $12 \mathrm{ppm}$. Due to the scale of the other plant's operation, as well as an unknown composition of their waste, determining the recovery and purity of the waste using this alternative poses a challenge. However, processing additional waste would reduce virgin NMP usage at the other plant by $180,000 \mathrm{~kg} /$ year. Relevant flowrates and compositions for this recovery system can be found in Table 3.

The life cycle emissions associated with the base case and all three recovery options are shown in Figure 2. Solvent extraction at the sister plant generates 464,000 and 435,000 kg/year of total and $\mathrm{CO}_{2}$ life cycle emissions, respectively. Compared to the base case, this results in a $62 \%$ reduction in total life cycle emissions and a $60 \%$ reduction in $\mathrm{CO}_{2}$ life cycle emissions. Compared to the distillation recovery alternative, extraction at the sister plant results in reductions of 32\%, and 33\% for total and life cycle $\mathrm{CO}_{2}$ emissions, respectively. These numbers are very similar to those for the on-site recovery option. Where emissions are avoided by removing the second distillation column, new emissions are introduced due to transportation of the waste from Parlin to the other plant.

The ReCiPe endpoint single scores (Figure 3) show that extraction and reuse of NMP at the sister plant results in single scores of 21,700, 10,300 and 19,100 Pt/year for human health, ecosystems, and resources, respectively. This results in reductions of $45 \%, 52 \%$, and $45 \%$ for human health, ecosystems, and resources, respectively, compared to the base case, and reductions of $0 \%, 18 \%$, and $25 \%$ for human health, ecosystems, and resources, respectively, compared to the distillation recovery design. Significant reductions in steam usage are slightly offset by production of the make-up chloroform and transportation of the waste from one plant to the other.

The life cycle operating costs of the base case and three alternatives can be found in Figure 4. Solvent extraction and reuse of NMP at the sister plant has a life cycle operating cost of $289,000 \$$ /year. Compared to the base case, operating costs are reduced by $1,177,000 \$ /$ year or $80 \%$. Extraction and reuse at the sister plant is slightly more expensive than on-site extraction and on-site reuse due to shipping costs, but a complete economic analysis shows that recovery and reuse at the sister plant is much more favorable in the long run; the main reason for this being the absence of any capital costs. Additionally, recovery and reuse at the sister plant has a higher operating cost for the Parlin facility, because they are still required to purchase virgin NMP for use in the resin precursor manufacture process. Also, it is unknown whether Parlin with pay the other plant (for hazardous waste "disposal") or vice versa (purchase of recoverable NMP); regardless of any transaction between the two plants, the overall operating cost within the DuPont Corporation is reduced by the NMP recovered and reused at the other plant as transactions between plants are within the system boundaries. Similarly, reductions in life cycle emissions and damage scores are for the DuPont Corporation, not for the Parlin facility.

As stated earlier, the sister plant already has the means to recover NMP using a solvent extraction system. Therefore, no equipment needs to be purchased or installed. Using the same methods as those used by Pastore et al. [2], the 5-year and 10-year NPV were calculated. The NPV after 5 years for solvent recovery and reuse at the sister plant was $\$ 2,923,000$ and $\$ 4,198,000$ after 10 years (Table 2). Over the course of 5 years, this alternative economically outperforms the distillation recovery option by $\$ 1,173,000$ and over the course of 10 years by $\$ 1,070,000$. 
By eliminating capital expenses, the NPV of the recovery of NMP dramatically improves.

\section{Conclusions}

An analysis of low-energy alternatives to distillation for the recovery of NMP from resin precursor manufacture process waste showed that recovery and reuse at the sister plant with an existing solvent recovery system would be both environmentally and economically beneficial. The use of publicly available TRI data facilitated identification of an outside plant that had a need for purified NMP waste. Further investigation indicated lower purity standards for recovered NMP at the sister plant. Ultimately, such a facility eliminates capital costs, while reducing operating costs, emissions, and environmental impact. Recovery and reuse using solvent extraction at the sister plant would save $\$ 4,198,000$ after 10 years, while reducing total life cycle and life cycle $\mathrm{CO}_{2}$ emissions by $62 \%$, and $60 \%$, respectively. In addition to this, damage scores were reduced by $45 \%, 52 \%$ and $45 \%$ in the human health, ecosystems, and resources categories, respectively. The results achieved through the use of solvent extraction and solvent reuse at the sister plant as an alternative to distillation and on-site solvent reuse are promising and show potential in solvent recovery applications throughout the fine/specialty chemical sector. Compared to distillation, use of existing solvent extraction equipment with underutilized capacity significantly increases the amount of money saved year after year and reduces emissions and environmental impact.

More broadly, the solvent recovery alternatives described have the potential to significantly reduce the generation of solvent waste throughout the entire specialty chemical sector, which, as mentioned in the introduction, was responsible for over 2 billion $\mathrm{kg}$ of waste in 2014 [6]. This study highlights the need for corporatewide solvent recovery plans. With such plans in place, other plants within the corporation can remain informed about solvent recovery efforts and be offered opportunities to pool capital resources to acquire solvent recovery systems, or as in this case, use existing solvent recovery process equipment for the benefit of two or more plants. More effective communication among plants would make it easy to determine in which cases solvents can be recovered at lower purities for reuse in processes that do not require high-purity solvents. This reduces the cost of recovery as well as emissions and the environmental impact associated with additional processing required for high-purity recovery.
Acknowledgments: The authors acknowledge the support of the U.S. Environmental Protection agency through the Pollution Prevention grant program (NP96291314-0). The authors also appreciate the support of the DuPont Corporation and Michael Hatton Jr., Charles Richwine, Jeffery Staniszewski (ret), and Nancy Uff of DuPont.

Conflict of interest statement: The authors declare to have no conflicts of interest regarding this article.

\section{References}

[1] Ghosh, MK, Mittal, KL, Eds., Polyimides: Fundamentals and Applications, Marcel Dekker: New York, 1996.

[2] Pastore BM, Savelski MJ, Slater CS, Richetti FA. Clean Technol. Environ. Policy 2016, 18, 2635-2647.

[3] Slater, CS, Savelski, MJ, Carole, WA, Constable DJ. In Green Chemistry in the Pharmaceutical Industry, Dunn, PJ, Wells, AS, Williams, MT, Eds., Wiley-VCH: Weinheim, 2010.

[4] Sehgal A. ALTSEP: Initial Steps on the Road to Low EnergyIntensity Chemical Separation, 20th Annual Green Chemistry \& Engineering Conference: Portland, 2016.

[5] Slater CS, Savelski MJ. J. Environ. Sci. Health, Part A: Toxic/Hazard. Subst. Environ. Eng. 2007, 42, 1595-1605.

[6] US Environmental Protection Agency, Toxics Release Inventory Program (US Environmental Protection Agency, 2016) https:// www.epa.gov/toxics-release-inventory-tri-program, Accessed July 2016.

[7] Sheldon RA. Chem. Soc. Rev. 2011, 41, 1437-1451.

[8] Slater CS, Savelski M). Innovations Pharm. Technol. 2009, 29, 78-83.

[9] Raymond MJ, Slater CS, Savelski MJ. Green Chem. 2010, 12, 1826-1834.

[10] Jiménez-González C, Constable DJC, Eds., Green chemistry and engineering: a practical design approach, 1st ed., Wiley: New York, 2011.

[11] Slater CS, Savelski MJ, Hounsell G, Pilipauskas D, Urbanski F. Clean Technol. Environ. Policy 2012, 12, 687-698.

[12] Slater CS, Savelski MJ, Moroz TM, Raymond MJ. Green Chem. Lett. Rev. 2012, 5, 55-64.

[13] Henderson RK, Jimenez-Gonzalez C, Constable DJ, Alston SR, Inglish GGA, Fisher G, Sherwood J, Binks SP, Curzons AD. Green Chem. 2011, 13, 854-862.

[14] Constable DJ, Jimenez-Gonzalez C, Henderson RK. Org. Process Res. Dev. 2007, 11, 133-137.

[15] Liaw DJ, Liaw BY. Macromol. Symp. 1997, 122, 343-348.

[16] Liou GS, Hsiao SH, Ishida M, Kakimoto M, Imai Y. J. Polym. Sci., Part A-1: Polym. Chem. 2002, 40, 2810-2818.

[17] Hergenrother PM. High Perform. Polym. 2003, 15, 3-45.

[18] Madbouly SA, Otaigbe JU. Prog. Polym. Sci. 2009, 34, 1283-1332.

[19] Razali M, Kim JF, Attfield M, Budd PM, Drioli E, Lee YM, Szekely G. Green Chem. 2015, 17, 5196-5205.

[20] Reisch M. Chem. Eng. News 2008, 86, 32.

[21] Constable DJ, Dunn PJ, Hayler JD, Humphrey GR, Leazer JL, Linderman RJ, Lorenz K, Manley J, Pearlman BA, Wells A, Zaks A, Zhang TY. Green Chem. 2007, 9, 411-420. 
[22] Alfonsi K, Colberg J, Dunn PJ, Fevig T, Jennings S, Johnson TA, Kleine HP, Knight C, Nagy MA, Perry DA, Stefaniak M. Green Chem. 2007, 10, 31-36.

[23] Jessop PG. Green Chem. 2011, 13, 1391-1398.

[24] Pollet P, Eckert CA, Liotta CL. Chem. Sci. 2011, 2, 609-614.

[25] Ji P, Atherton J, Page MI. J. Org. Chem. 2011, 76, 1425-1435.

[26] National Research Council. Sustainability in the Chemical Industry: Grand Challenges and Research Needs - A Workshop Report, The National Academies Press: Washington, 2005.

[27] Fang J, Zhao H, Qi J, Li C, Qi J, Guo J. Chin. J. Chem. Eng. 2015, 23, 934-940.

[28] Dejanovic I, Matijasevic Lj, Olujic Z. Chem. Eng. Process. 2010, 49, 559-580.

[29] Jana AK. Energy Convers. Manage. 2014, 77, 287-297.

[30] Panda, H, Ed., The complete guide on industrial pollution control, Asia Pacific Business Press Inc.: Delhi, 2011.

[31] Sato K, Sugimoto K, Shimotsuma N, Kikuchi T, Kyotani T, Kurata T. J. Membr. Sci. 2010, 409-410, 82-95.

[32] Das S, Banthia AK, Adhikari B. Desalination 2006, 197, 106-116.

[33] Goedkoop M, Heijungs R, Huijbregts M, De Schryver A, Struijs J, van Zelm. ReCiPe 2008: a life cycle impact assessment method which comprises harmonised category indicators at the midpoint and the endpoint level: report l: characterisation, Leiden University: Leiden, 2009.

[34] US Energy Information Administration, Electricity Data Browser (US Energy Information Administration, 2015) http://www.eia. gov/electricity/data/browser, Accessed June 2015.

[35] ICIS, Indicative Chemical prices (RBI, 2008) http://www.icis. com/chemicals/channel-info-chemicals-a-z, Accessed June, 2015.

[36] Towler, G, Sinnott, RK, Eds., Chemical engineering design, 2nd ed., Butterworth-Heinenmann: Oxford, 2013.

[37] Kirby, AJ, Ed., Polyimides: materials, processing, and applications, Pergamon Press Inc: Tarrytown, 1992.

[38] Ghosh UK, Pradhan NC, Adhikari B. J. Membr. Sci. 2006, 285, 249-257.

[39] Garcia-Chavez LY, Garsia CM, Schuur B, de Haan AB. Ind. Eng. Chem. Res. 2012, 51, 8293-8301.

[40] Stoffers M, Gorak A. Sep. Purif. Technol. 2013, 12, 415-422.

[41] Yang D, Zhu B. Huaxue Yanjiu Yu Yingyong 2010, 22, 1446-1449.

[42] Hansen, CM, Ed., Hansen solubility parameters, 2nd ed., CRC Press Inc: Boca Raton, 1999. 